Cumhuriyet International Journal of Education-CIJE

e-ISSN: 2147-1606

Vol 5 (2), 2016, $50-66$

\title{
Development of Perceived Parental Relationship Scale and Its Psychometric Properties
}

\author{
Summary
}

\section{INTRODUCTION}

The fact that a person chosen by an individual as a spouse shares similar characteristics with that individual's parents may affect the marital relationship between two people. It is even possible for an individual to have the tendency to choose a spouse sharing similar characteristic with his/her parents when looking for a person sharing similar characteristics with himself/herself, or vice versa. On the other hand, he/she might choose an authoritarian spouse while he/she is also of an authoritarian character. According to Rushton and Nicholson (1988), advocates of Buss and Barnes (1986) who claimed that many spouses exhibit similar characteristics, the similarity between individuals and their parents is a result of genetic affinity as individuals strive for ensuring their genes to survive through passing them on. Bereczkei (2004) further explains that the spouse one chooses shows resemblance with each one of his/her parents.

According to Davies and Cummings (1994) children in a family react to how their parents solve their relationship problems (conflicts) and take them as a model. Thus, emotions and thoughts of the children take form accordingly. Children will adopt the same pattern when their parents are able to solve their marital relationship problems successfully. This might indicate that an individual's behavior in his/her marital relationship will depend on the behavior observed in their parents' marital relationship.

It is widely believed that marital satisfaction is in close relation with an individual's positive perception of his/her parents' marital relationship in order for an individual to feel psychologically good about himself/herself and for the members of a family to be happy. It was reported that men tend to prefer women who resemble their mothers while women tend to prefer people who resemble their fathers (Roithmayer, 2011). Therefore, the role parents play in a marital relationship should be taken into consideration. In this context, our perception of the relationship behavior of our parents plays an important role in our choice of spouse, our relationship behavior and the relationship continuity. Literature review revealed that there are no studies analyzing the relation between the perceived parental relationship and subjects such as choice of spouse, similarity theories, taking parents as a model and self-esteem.

\section{PURPOSE}

The purpose of this study is to develop a measuring tool in order to evaluate the perceived paternal-marital relationship behavior.

\section{METHOD}

Specialists were sought for their advice on the content validity of the scale developed and AFA was conducted for the structural validity. Cronbach's Alpha was used in order to establish the reliability of the scale and corrected item total correlations of the items of the scale were analyzed for the item analysis.

\section{FINDINGS}

The results of the AFA revealed that both mother and father figures of the scale consist of 37 items and 5 factors (authoritarian, neglectful, protective, democratic, and dependent) each. When the factor loadings of the scale for both mother and father figures were assessed, it was found that factor loadings of the items were sorted between .56 and .89 
for the mother figure while they were sorted between .53 and .82 for the father figure. The coefficient of internal consistency for mother figure (authoritarian $=.80$, neglectful $=.88$, protective $=.83$, democratic $=.95$, and dependent $=.88$ ) and father figure (authoritarian $=.73$, neglectful $=.89$, protective $=.81$, democratic $=.95$, and dependent $=.86)$ indicates that the scale is a reliable measuring tool.

\section{DISCUSSION and CONCLUSIONS}

The factors obtained from the Exploratory Factor Analysis conducted for the structural validity were compared with the specialist opinion which revealed a consistency in the results. According to Büyüköztürk (2010) the total variance of the structure obtained as a result of the exploratory factor analysis needs to be higher than $41 \%$ and the factor loadings of the items listed under the factors need to be higher than .32 in order to establish the structural validity of a newly developed scale. In this context, according to the AFA results of the study, the factor loadings of the items of the scale were higher than .50 while mother figure accounted for $60.77 \%$ of the total variance and father figure accounted for $60.07 \%$ of the total variance. In the light of these findings, it can be said that the variance percentages and factor loadings of the items obtained from the AFA were sufficient for the structural validity.

The analyses conducted in order to determine the validity and reliability of the parental relationship behavior scale developed for the first time with this study involved data collected from a limited sample size and it is a cross-sectional study. Nevertheless, it was found that the sample was not representative of different ethnicities living in Turkey when the socio-demographic characteristics of the sample were evaluated. For this reason, the scale attempted to be developed in this study needs to be tested on samples with diverse socio-demographic characteristics and its validity and reliability analyses need to be assessed again. As developing a scale is a process, assessment and revision of the psychometrics using different samples in different periods of time may contribute to the measuring capacity of the scale. Test-retest method was not employed in this study for reliability purposes. Future research might consider the test-retest method in order to test the reliability of this scale. Nevertheless, it would be rewarding to investigate the relationship between the scale developed in this study and other reliable scales which measure other structures which may be related with perceived parental relationship behavior in order to establish the consistency of the scale. 